\title{
Analysis of aggregated tick returns: evidence for anomalous diffusion
}

\author{
Philipp Weber \\ Institut für Theoretische Physik, Universität zu Köln, 50937 Köln, Germany*
}

\begin{abstract}
In order to investigate the origin of large price fluctuations, we analyze stock price changes of ten frequently traded NASDAQ stocks in the year 2002. Though the influence of the trading frequency on the aggregate return in a certain time interval is important, it cannot alone explain the heavy tailed distribution of stock price changes. For this reason, we analyze intervals with a fixed number of trades in order to eliminate the influence of the trading frequency and investigate the relevance of other factors for the aggregate return. We show that in tick time the price follows a discrete diffusion process with a variable step width while the difference between the number of steps in positive and negative direction in an interval is Gaussian distributed. The step width is given by the return due to a single trade and is long-term correlated in tick time. Hence, its mean value can well characterize an interval of many trades and turns out to be an important determinant for large aggregate returns. We also present a statistical model reproducing the cumulative distribution of aggregate returns. For an accurate agreement with the empirical distribution, we also take into account asymmetries of the step widths in different directions together with crosscorrelations between these asymmetries and the mean step width as well as the signs of the steps.
\end{abstract}

The mechanics of stock price changes were studied already more than a hundred years ago, when Bachelier modelled price movements as a diffusion process with Gaussian fluctuations [1]. However, empirical studies show that the distribution of returns has heavy tails 2, 3, 4, 5, 6, 7, 8, 9, 10, 11, 12, 13], meaning that events with large price changes are much more probable than in a Gaussian distribution. In addition, the functional form of the distribution stays similar if the return is aggregated on very different time scales from seconds to months, approximating a Gaussian distribution only if the time scale becomes very large [2, 3].

These findings would suggest that stock returns have a Lévy stable distribution 2, 13, 14, 15. In a Lévy flight, the second moment would be divergent and extreme returns aggregated over a long time would be determined by very large price jumps on smaller time scales. However, empirical studies find evidence that the tail of the return distribution follows a power law with exponent around $\alpha-1=3$ so that it does not agree with the stable Paretian hypothesis 3, 4, 5, 6, 7, 16, 17, 18, 19, 20, 21].

The cause of the fat tails is currently a subject of great interest 22, 23, 24, 25, 26]. Farmer et al. find that the distribution of returns due to a single trade (tick returns) is similar to the distribution of returns aggregated on longer time scales with the same tail exponent [25]. Although the tail exponent is outside the Lévy regime $0<\alpha-1<2$, they argue that similar to a Lévy flight both distributions are caused by the same microscopic mechanism, so that large aggregate returns are due to single exceptionally large tick returns. Plerou et al. describe the price movements as a diffusion process with a fluctuating diffusion constant and relate the distribution of aggregate returns to the distribution of the variance of the tick returns 26].

*Electronic address: pw@thp.uni-koeln.de
In the present paper, we investigate the transition from tick returns to returns aggregated in intervals with a larger number of trades. It is well documented (e.g. in 27, 28]) that the number of trades in a time interval is an important determinant of the aggregate return. However, the trading frequency alone cannot account for the observed fat tailed distribution of aggregate returns [26]. Thus, we remove the direct influence of the trading frequency by analyzing intervals with a constant number of trades so that effects due to other quantities like the tick return size are more clearly visible.

We study how each aggregate return is actually built from the basic quantities involved in the process, and thus examine the mechanism leading to large price fluctuations. According to the central limit theorem, independent tick returns would in aggregation lead to Gaussian distributed returns. However, we find that the tick return size is long-term correlated in tick time (compare 29, 30, 31, 32, 33, 34, 35, 36, 37]), so that the conditions of the central limit theorem are not fulfilled. Thus, the mean tick return size can well characterize an interval of many trades and its fluctuations lead to the non-Gaussian behavior of the aggregate return. In this picture, large aggregate returns do not occur because of a few very large tick returns, but rather when the average tick return is large, so that even Gaussian fluctuations in the direction of the trades can lead to aggregate return larger than in a Gaussian distribution.

The remainder of this paper is organized as follows: section I shows our model for the price diffusion process, in section II we describe the data set used for this study, section III shows the influence of the tick return size on the aggregate return while section IV focusses on the influence of differences in the direction of tick returns (number difference). Section V compares the number difference and the flow of market orders and in section VI we present a statistical model which approximates the distribution of aggregate returns. We conclude with a discussion of our results in section VII. 


\section{MODEL}

We study intervals with a fixed number of $N=100$ trades. If the price of a stock before the $i$-th trade is $s_{i}$, we define the return due to a single trade, the tick return, as

$$
\delta g_{i}=\ln \left(s_{i+1}\right)-\ln \left(s_{i}\right)
$$

The interval $I_{j}$ contains all $N$ trades with index $i$ between $j N$ and $(j+1) N$, so the aggregate return $G_{j}$ is given by the sum over all $\delta g_{i}$ with $i \epsilon I_{j}$ :

$$
G_{j}=\sum_{i \in I_{j}} \delta g_{i}
$$

We want to discuss two special cases in order to analyze the mechanism leading to large aggregate returns $G_{j}$. In the first case, $G_{j}$ is dominated by one (or a few) extremely large $\delta g_{i_{0}}^{\max }$, so that

$$
G_{j}=\delta g_{i_{0}}^{\max }+\sum_{i \in I_{j}, i \neq i_{0}} \delta g_{i} \approx \delta g_{i_{0}}^{\max } .
$$

Thus, $G_{j}$ becomes large if $\delta g_{i_{0}}^{\max }$ is exceptionally large.

In the second case, we assume that there is no extremely large tick return dominating the aggregate return, so that we focus on the average size $\Delta g_{j}$ of the non-zero tick returns, which is defined by

$$
\Delta g_{j}=\frac{1}{n_{j}} \sum_{\delta g_{i} \neq 0, i \epsilon I_{j}}\left|\delta g_{i}\right|
$$

Here, $n_{j}$ is the number of $\delta g_{i} \neq 0$ in the interval $I_{j}$. Neglecting assymetries in the $\delta g_{i}$, we can replace all $\delta g_{i} \neq$ 0 by $\operatorname{sign}\left(\delta g_{i}\right) \Delta g_{j}$ and approximate the aggregate return by

$$
G_{j} \approx \Delta g_{j} \sum_{\delta g_{i} \neq 0, i \in I_{j}} \operatorname{sign}\left(\delta \mathrm{g}_{\mathrm{i}}\right)=\Delta \mathrm{g}_{\mathrm{j}} \Delta \mathrm{N}_{\mathrm{j}}
$$

where $\Delta N_{j}=\sum_{\delta g_{i} \neq 0, i \in I_{j}} \operatorname{sign}\left(\delta \mathrm{g}_{\mathrm{i}}\right)$ is called number difference. Similarly, $G_{j}$ can be described as a diffusion process with

$$
\left\langle G_{j}^{2}\right\rangle \approx D_{j} N
$$

where the diffusion constant $D_{j}=\frac{n_{j}}{N} \Delta g_{j}^{2}$ varies due to the varying step width $\Delta g_{j}$ and the number $n_{j}$ of nonzero tick returns.

In the approximation given by Eq. [5] we can study the influence of the mean size of the tick returns as well as asymmetries in their direction. A large aggregate return can occur if the price moves more often in one direction than in the other. Thus, with large temporary correlations between the signs, even small tick returns could compose a large $G_{j}$. On the other hand, if $\Delta g_{j}$ is larger, even a small asymmetry in the signs can lead to a large return.
The two approximations given in Eq. 3] and Eq. 5] are analyzed in sections III and IV of this paper, but in section VI we also consider the error term neglected in Eq. 5 . An exact formulation writes

$$
G_{j}=\Delta g_{j} \Delta N_{j}+\frac{2 n_{j}^{+} n_{j}^{-}}{n_{j}}\left(\Delta g_{j}^{+}-\Delta g_{j}^{-}\right)
$$

where $\Delta g_{j}^{+}$and $\Delta g_{j}^{-}$are the average tick returns in positive and negative direction while $n_{j}^{+}$and $n_{j}^{-}$are the numbers of non-negative tick returns in positive and negative direction.

\section{DATA ANALYSIS}

We analyzed order book data of the year 2002 from Island ECN for the ten most frequently traded stocks 38]. Since the Island ECN is a secondary market where only part of the whole stock volume is traded, we also studied the index fund QQQ which was mainly traded via Island until September 2002. Since our results for the ten stocks and QQQ are similar, we find no evidence that secondary market characteristics of Island affect our analysis negatively.

In an electronic market place like Island, people can place limit orders to buy or to sell at a given or better price (limit price), which is specified in the order. These orders are stored in the order book and they are only executed when the actual stock price reaches the limit price. A trade is initiated by a market order indicating that someone wants to buy or sell immediately at the best available price. Such a market order executes the limit orders offering the best prices until the number of shares specified in the market order is traded.

Our dataset contains information about every limit order so that we are able to reproduce the market situation at each instant of time. We combine those limit order executions with identical time stamps as they reflect the same market order. Therefore, we can analyze the impact of each single market order on the price. In this analysis, the price is defined as the mid-quote price which is the mean of the best available buy (bid) and sell (ask) limit prices (quotes). We study intervals with a fixed number of $N=100$ market orders and have approximately 100,000 intervals in our dataset for ten stocks. Thus, on average a 100 trade interval corresponds to about 10 minutes, but the trading frequency fluctuates strongly so that 100 trades can correspond to time intervals with very different lengths.

We determine the mid-quote price $s_{i}$ just before the execution of the $i$-th market order. Since most trades change the price just by the size of the gap between the best and the second best limit price 25], the tick return $\delta g_{i}$ corresponds to the gap size. We note that the price can (and often does) change between two consecutive market orders due to placement or cancellation of limit orders so that $\delta g_{i}$ does not provide a direct estimate 


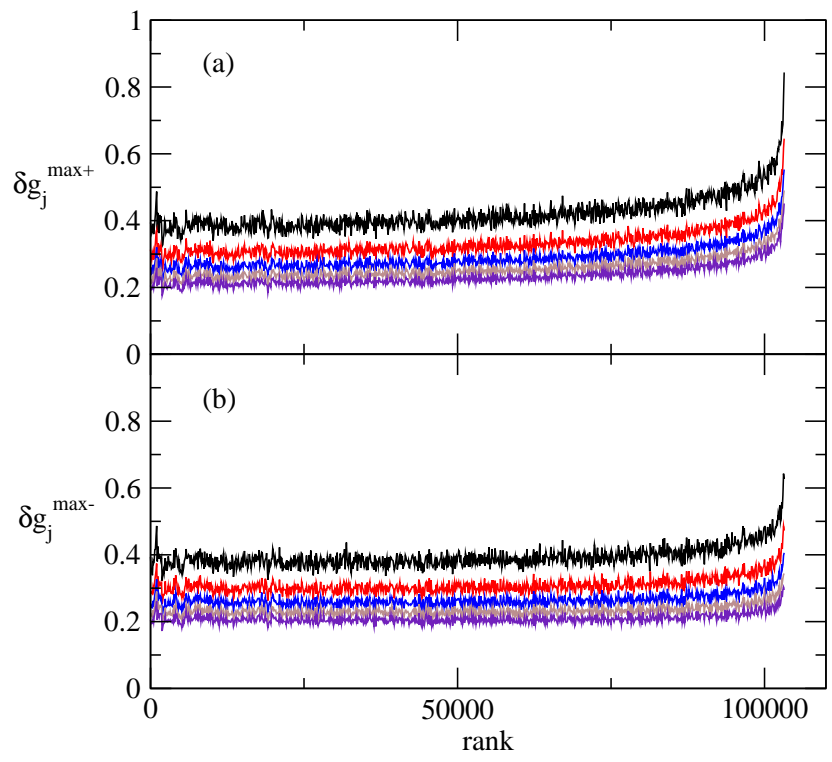

FIG. 1: (Color online) Five largest price changes (a) $\delta g_{j}^{\max +}$ and (b) $\delta g_{j}^{\max -}$ due to a single trade with (a) the same and (b) the opposite sign as the aggregate return in that 100 tick interval, plotted against the rank of the corresponding aggregate return $\left|G_{j}\right|$ for the combined data of ten Nasdaq stocks in 2002 (smoothed by averaging over 100 intervals). For large $\left|G_{j}\right|$, the size of the $\delta g_{j}^{\max }+$ increases by a factor of two while the increase in the $\delta g_{j}^{\max -}$ is slightly smaller. The sum over all five $\delta g_{j}^{\max +}$ reaches more than 3 standard deviations for intervals with extremely large $\left|G_{j}\right|$, but the fluctuations in the opposite direction are almost equally large.

of the gap size. We normalize the tick returns $\delta g_{i}$ by the standard deviation of the aggregate return $G_{j}$ for each stock individually so that we can combine the results for different stocks.

\section{INFLUENCE OF THE SIZE OF TICK RETURNS}

First, we investigate the question whether large tick returns caused by large gaps in the order book can be responsible for large aggregate returns. To this end, we start with the approximation shown in Eq. 3 where a few extremely large tick returns (corresponding to some very large gaps in the order book) lead to a very large aggregate return $G_{j}$. In order to test this hypothesis, we analyzed the five largest tick returns $\delta g_{j}^{\max +}$ with the same sign as the aggregate return $G_{j}$ (i.e. the five largest positive $\delta g_{i}$ if $G_{j}>0$ and the five largest negative $\delta g_{i}$ for $\left.G_{j}<0\right)$ in each time interval. To this end, we sort the intervals by $\left|G_{j}\right|$ and plot the $\delta g_{j}^{\max +}$ against the rank of the interval according to its return $\left|G_{j}\right|$.

Fig. 1(a) shows the values of these $\delta g_{j}^{\max }$ in intervals with small $G_{j} \approx 0$ on the left while the values for large returns exceeding five standard deviations can be found on the right. Since there are large fluctuations

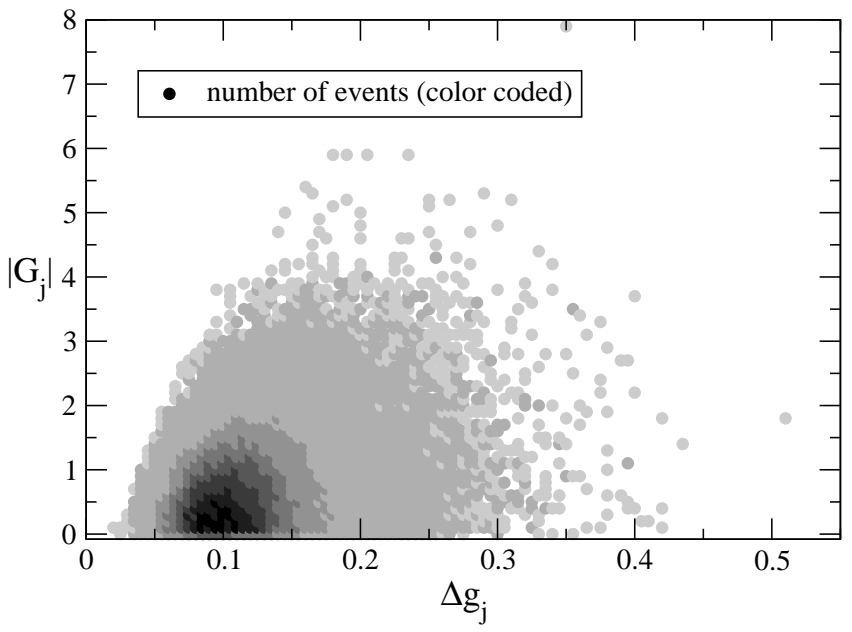

FIG. 2: Density plot of the 100-trade-return $\left|G_{j}\right|$ of ten Nasdaq stocks against the average return of a single trade $\Delta g_{j}$ for each interval. The Points are coded from light grey to black indicating the number of events from 1 to more than 500. A linear regression has only a small correlation coefficient $R^{2}=0.07$.

in the data, we smoothed the curves by averaging over 100 intervals. The $\delta g_{j}^{\max +}$ grow by a factor of two between small and very large returns $\left|G_{j}\right|$. When aggregated, these five largest $\delta g_{j}^{\max +}$ can reach about three standard deviations, which is almost half of the largest aggregate returns.

In Fig. I(b), we plot the five largest tick returns $\delta g_{j}^{\max -}$ with the opposite direction as the aggregate return against their rank. The $\delta g_{j}^{\max -}$ behave similarly to the $\delta g_{j}^{\max +}$, though the increase for large aggregate returns is slightly weaker. However, even for the largest aggregate returns the difference between the $\delta g_{j}^{\max +}$ and $\delta g_{j}^{\max -}$ is rather small, so that there are also large tick returns reducing the aggregate return.

Our results suggest that large aggregate returns are not the result of single exceptionally large tick returns since very large tick returns occur in both directions and cancel each other out. In the following, we want to focus not on the extreme tick returns, but on the influence of their mean value. More precisely, we analyze Eq. [5 and the mean tick return $\Delta g_{j}$ of all non-zero $\left|\delta g_{i}\right|$ in the interval $I_{j}$ as defined in Eq. 4. A density plot of $\left|G_{j}\right|$ against $\Delta g_{j}$ is shown in Fig. 2] It seems that extremely large returns $G_{j}$ correspond to larger average tick returns $\Delta g_{j}$, but the broad distribution suggests that the explanatory power of $\Delta g_{j}$ alone for the aggregate return $G_{j}$ is small, which is confirmed by the low correlation coefficient $R^{2}=0.07$ of a linear regression.

In order to clarify the relation between the extreme values of $\left|G_{j}\right|$ and $\Delta g_{j}$, we sort the intervals by $\left|G_{j}\right|$ and plot $\Delta g_{j}$ against the rank of the interval according to its return $\left|G_{j}\right|$. In Fig. [3 (black curve), we see that large returns $\left|G_{j}\right|$ coincide with larger tick returns as $\Delta g_{j}$ changes by a factor of two from very low aggregate re- 


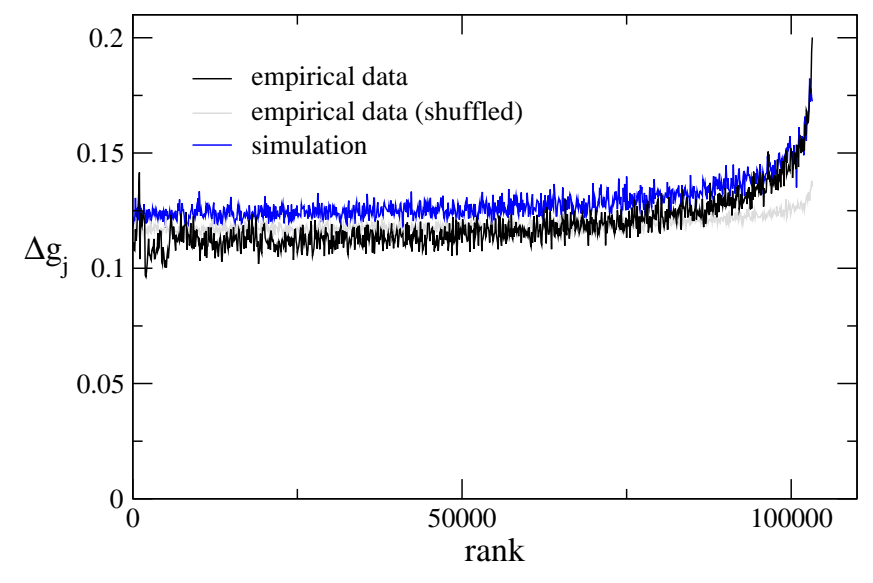

FIG. 3: (Color online) Black curve: average tick return $\Delta g_{j}$ of ten Nasdaq stocks plotted against the rank of the corresponding aggregate return $\left|G_{j}\right|$, smoothed by averaging over 100 intervals. Going from the smallest returns $\left|G_{j}\right| \approx 0$ to returns larger than 5 standard deviations, $\Delta g_{j}$ increases by a factor of two. Light grey curve: after shuffling the tick returns for each stock, the same curve is only slightly increased for the largest aggregate returns, the effect is much smaller than for the original data. Blue curve (or dark grey): the simulation according to the statistical model discussed in section VI shows a similar behaviour as the empirical data, but in the simulation $\Delta g_{j}$ is a little larger than the empirical one except for the largest $\left|G_{j}\right|$ where the simulated $\Delta g_{j}$ is slightly smaller than the empirical one.

turns to large returns of several standard deviations. In comparison with the largest tick returns $\delta g_{j}^{\max }+$ shown in Fig. 11 the change of a factor of two is similar, but the mean $\Delta g_{j}$ is two to four times smaller than the largest tick returns.

This finding can be explained by the presence of autocorrelations in the time series of $\delta g_{i}$, which can be illustrated when we shuffle the data for each stock by exchanging each tick return with another randomly chosen tick return. The light grey curve in Fig. 3 shows that for shuffled data $\Delta g_{j}$ increases only marginally for large aggregate returns, suggesting that autocorrelations of the tick returns have a strong influence on the mean tick return size $\Delta g_{j}$. Indeed, we find that the absolute values $\left|\delta g_{i}\right|$ of the tick return are long-range correlated in tick time with a correlation function decaying like $\Delta i^{-0.16}$ for large time lags $\Delta i=\left|i_{1}-i_{2}\right|$, as shown in Fig. 4. If these correlations are destroyed by shuffling, in each interval of 100 trades only a few large tick returns remain so that the average over these 100 tick returns approximates the global mean of all tick returns in the data set.

In contrast, in the empirical unshuffled data correlations lead to intervals where many tick returns are large, so that the average tick return size is also large. The average tick return size $\Delta g_{j}$ can well characterize the interval only because these autocorrelations exist. It turns out that the increase of $\Delta g_{j}$ by a factor of two is the main effect where the original empirical data deviates significantly from shuffled data. Hence, we suggest that

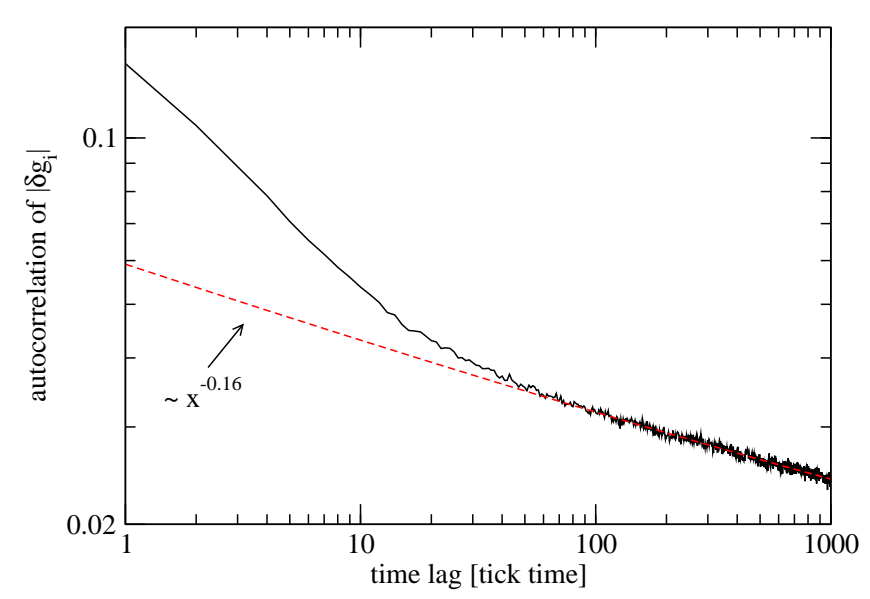

FIG. 4: Autocorrelation function of the absolute value of the tick return $\left|\delta g_{i}\right|$ averaged over the data of ten Nasdaq stocks in 2002. The function shows a power law decay in tick time proportional to $\Delta i^{-0.16}$ for large $\Delta i$.

fluctuations of the tick return size are responsible for the non-Gaussian fluctuations of the aggregate return.

Using Eq. 5. we can estimate whether the change by a factor of two of the average tick return alone is enough to explain large aggregate returns $G_{j}$ of more than five standard deviations. To this end, we focus on the intervals with the 50 largest aggregate returns ranging from approximately 4 to almost 8 standard deviations. Here, we find that $\Delta g_{j}$ fluctuates between 0.14 and 0.35 . Assuming uncorrelated returns, $\Delta N_{j}$ should be of the order $\sqrt{N} \approx 10$ if each trade would lead to a price change, but normal fluctuations could well lead to $\Delta N_{j}$ twice as large as $\sqrt{N}$, so that large tick returns together with fluctuations in the number difference could explain the large aggregate returns we find in our data set.

Thus, we find that in intervals with 100 trades large $\left|G_{j}\right|$ do not mainly depend on single extremely large tick returns. It rather turns out that correlations between the tick returns lead to large average tick returns $\Delta g_{j}$ in an interval, and the fluctuations of $\Delta g_{j}$ can account for the non-Gaussian distribution of the aggregate returns.

\section{NUMBER DIFFERENCE}

The diffusion process of aggregate returns is not only influenced by the step width (i.e. the tick return size), but also by the direction of the steps. Therefore, we now analyze the influence of the number difference $\Delta N_{j}$ in Eq. [5] In order to treat positive and negative aggregate returns in the same analysis, it is useful to replace $\Delta N_{j}$ by the sign-adapted number difference

$$
\Delta n_{j}=\operatorname{sign}\left(\mathrm{G}_{\mathrm{j}}\right) \Delta \mathrm{N}_{\mathrm{j}} .
$$

A positive value of $\Delta n_{j}$ indicates that the price tends to move in one specific direction leading to an aggregate return with the same sign. $\Delta n_{j}$ can be negative if there are 


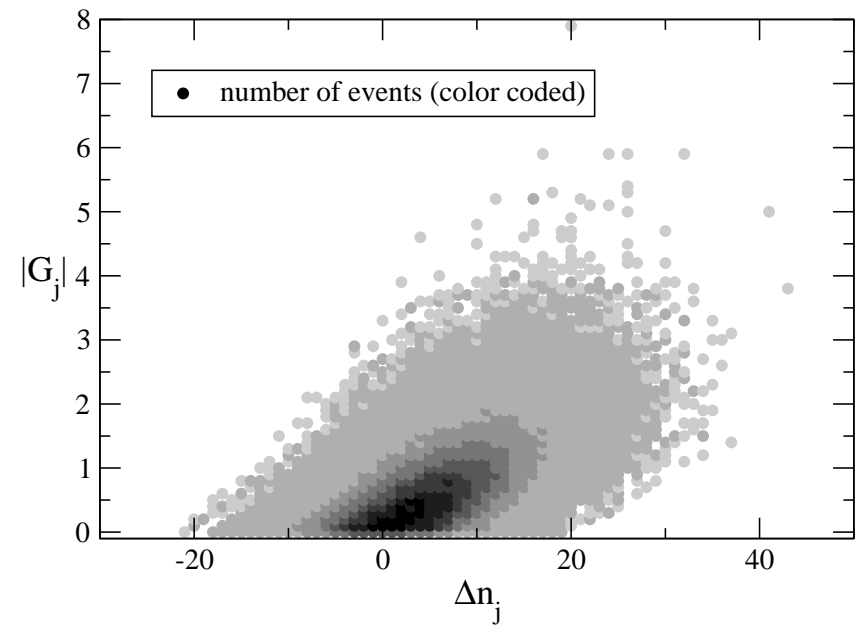

FIG. 5: Density plot of the aggregate return $\left|G_{j}\right|$ against the difference $\Delta n_{j}$ between the number of tick returns with the same and with the opposite direction as the aggregate return, for ten Nasdaq stocks. The points are coded from light grey to black indicating the number of events from 1 to more than 600. A linear regression has a large correlation coefficient $R^{2}=0.32$.

a few large tick returns determining the direction of the aggregate return, but also many small tick returns with the opposite direction which do not affect the aggregate return very much. Fig. [5 shows a density plot of the aggregate return $\left|G_{j}\right|$ against the sign-adapted number difference $\Delta n_{j}$. A linear regression yields an $R^{2}$ of 0.32 , a large correlation coefficient confirming the visual impression that $\Delta n_{j}$ and $\left|G_{j}\right|$ are strongly connected. We can also see that $\Delta n_{j}$ is mostly positive for large returns $G_{j}$, so that each large price change is accompanied by a certain sign-adapted number difference $\Delta n_{j}$.

We now plot in Fig. [6 $\Delta n_{j}$ against the rank according to $\left|G_{j}\right|$. We find that except for the largest (approximately $15 \%$ ) of the aggregate returns, $\Delta n_{j}$ grows linearly with the rank while in Fig. [3 $\Delta g_{j}$ remained almost constant in that region. For the largest ranks, $\Delta n$ increases more rapidly, so that all in all the smoothed curve (averaged over 100 intervals) grows from 0 to 18 between very small and extremely large aggregate returns. Thus, in intervals with very large returns there are approximately 18 trades pushing the price in one direction (assuming that all other trades cancel each other out), so that even with rather small tick returns this can lead to large returns in aggregation. Focusing on the 50 largest $G_{j}$, we find that $\Delta n_{j}$ ranges from 4 to 41 , clearly above the expected standard deviation of 10 when assuming uncorrelated returns and $n_{j}=N$.

Thus, the fluctuations of $\Delta n_{j}$ around the mean value are crucial for getting large aggregate returns. The number difference seems to be the main mechanism affecting the aggregate return since it changes much more drastically than the tick return size when the aggregate return increases. On the other hand, when we compare the re-

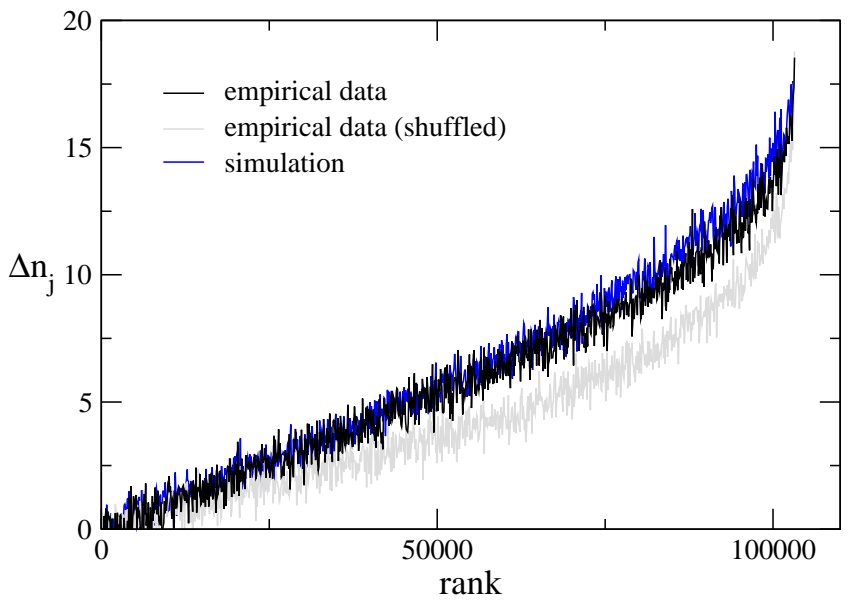

FIG. 6: (Color online) Black curve: the sign-adapted number difference $\Delta n_{j}$ is plotted against the rank according to the aggregate return $\left|G_{j}\right|$ for 10 Nasdaq stocks, smoothed by averaging over 100 intervals. $\Delta n_{j}$ grows from zero to 18 . The relation between $\Delta n_{j}$ and the rank seems to be linear except for the largest $15 \%$ of the aggregate returns. A simulation (blue curve (or dark grey)) using a normal distribution for $\Delta N_{j}$ leads to nearly the same dependance on the rank. For shuffled data (light grey curve), the curve is slightly flatter, but the difference is not large.

sults to the analysis with shuffled data (light grey curve in Fig. [6), it turns out that this effect is very similar to what happens with random price changes. Hence, the basic movement of the aggregate return seems to depend mostly on the number difference, but the non-Gaussian large aggregate price changes only occur if the tick returns are large.

\section{MARKET ORDER SIGNS AND DIRECTION OF TICK RETURNS}

It is known that the signs of market orders are strongly correlated [39, 40] which means that there is a large probability that a buy market order will be followed by another buy market order. Thus, it is probable that large number differences in the direction of tick returns are caused by large numbers of equally signed market orders. In order to analyze the relation between the number difference and the market order flow, we define the difference $\Delta n_{j}^{m}$ between the number $n_{j}^{m+}$ of market orders with the same direction as $G_{j}$ and the market orders with opposite direction $n_{j}^{m-}$ :

$$
\Delta n_{j}^{m}=n_{j}^{m+}-n_{j}^{m-} .
$$

In Fig. 7 we plot the sign-adapted number difference $\Delta n_{j}$ against the market order difference $\Delta n_{j}^{m}$. We find a strong correlation between $\Delta n_{j}$ and $\Delta n_{j}^{m}$, a linear regression yields a correlation coefficient $R^{2}$ of 0.29 . However, 


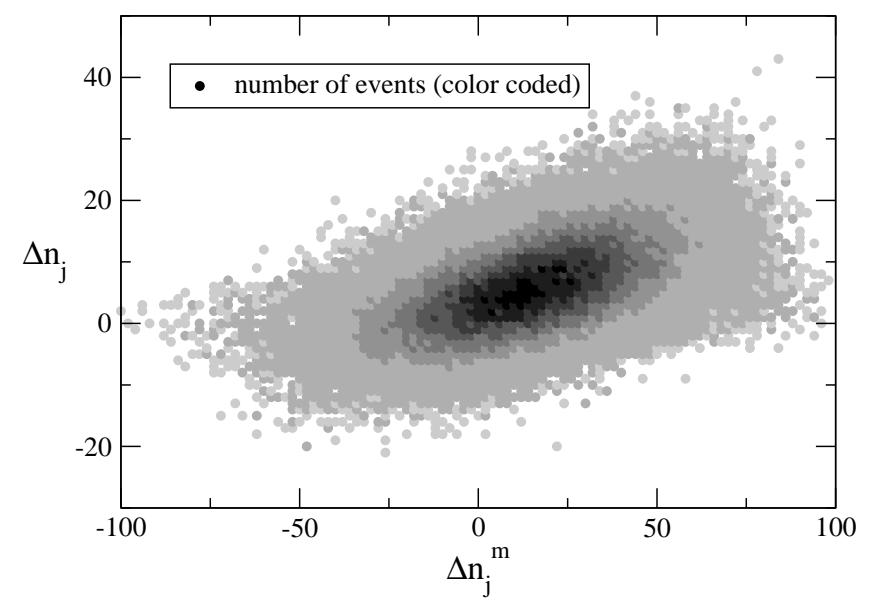

FIG. 7: Comparison between sign-adapted number difference $\Delta n_{j}$ and market order difference $\Delta n_{j}^{m}$ for ten Nasdaq stocks. The Points are coded from light grey to black indicating the number of events from 1 to more than 200. The correlation coefficient of a linear regression yields $R^{2}=0.29$, thus there is a strong connection between the two quantities. On the other hand, the events scatter widely so that small $\Delta n$ are often linked with large $\Delta n_{j}^{m}$ and vice versa.

there are also large fluctuations suggesting that the number difference is also due to order book dynamics, namely limit order placement and cancellation as well as asymmetries in the order book. A model for price formation due to these quantities was recently proposed by Mike and Farmer [41].

\section{DISTRIBUTION OF AGGREGATE RETURNS AND A STATISTICAL MODEL}

In the first part of this paper, we analyzed the mechanism leading to large aggregate returns and showed that the varying step width $\Delta g_{j}$ accounts for the nonGaussian behavior of the diffusion process of price movements. Now we want to use our results in a statistical model and reproduce the cumulative distribution function of the absolute value of the aggregate return $\left|G_{j}\right|$.

The model given by Eq. 5 belongs to the well-known class of stochastic volatility models (see e.g. 42]) consisting of a noise term multiplied by a time-dependent volatility giving the magnitude of the fluctuations. In the present paper, the model is based on a microscopic description of the price process, so that we can fit the microscopic quantities determining the aggregate return in order to estimate the parameters of the model. In this approach the model is parameter-free in the sense that there are no parameters fitting the aggregate returns directly, though we fit the distributions of its determinants like the step width $\Delta g_{j}$ and the number difference $\Delta N_{j}$. We also discuss corrections to the model by including the tick return asymmetries according to Eq. [7]

We first analyze the distributions of $\Delta g_{j}$ and $\Delta N_{j}$.

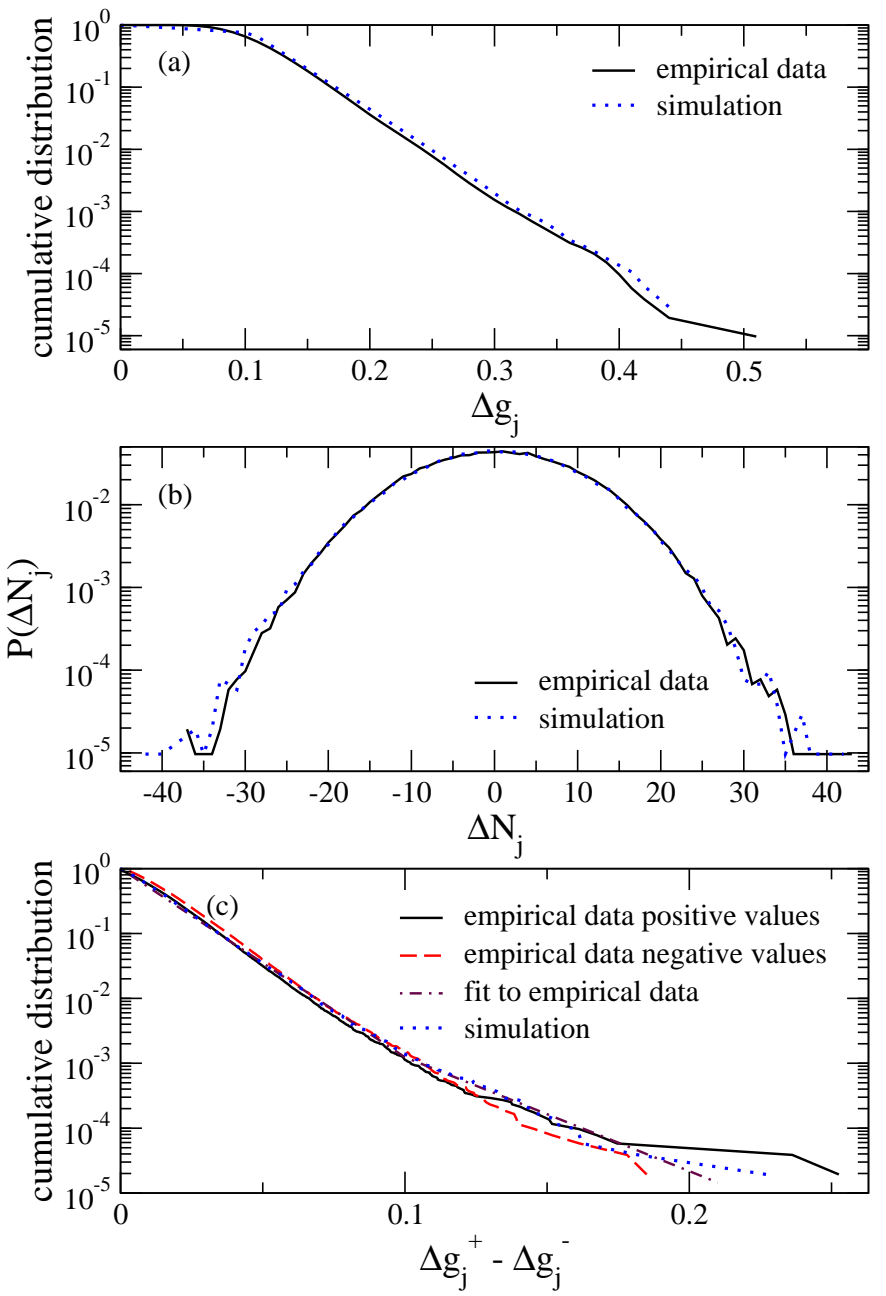

FIG. 8: (Color online) Estimation of the parameters for the simulation (results shown as dotted lines) from empirical data for ten Nasdaq stocks. (a) The tail of the cumulative distribution of $\Delta g_{j}$ (line) can be well fitted with $P\left(x>\Delta g_{j}\right)=e^{-a\left(x-x_{0}\right) / \Delta \bar{g}}$ where $\Delta \bar{g} \approx 0.12$ is the average of all $\Delta g_{j}$ and the parameters are $a=3.6$ and $x_{0}=0.094$. For $\Delta g_{j} \lesssim x_{0}$ the limited tick size leads to a plateau. (b) The probability distribution of $\Delta N_{j}$ (line) follows in good approximation a normal distribution with mean 0.24 and standard deviation 9.0. (c) As a rough approximation, the average of the cumulative distribution of the positive (line) and negative (dashed line) values of $\Delta g_{j}^{+}-\Delta g_{j}^{-}$are parameterized proportional to two exponential functions $e^{-a_{1,2} x / \Delta \bar{g}}$ for $\left|\Delta g_{j}^{+}-\Delta g_{j}^{-}\right| \lessgtr 0.1$, with $a_{1}=8.0$ and $a_{2}=4.8$ (dashdotted line). The simulation (dotted line) uses the adapted $a_{1}=9.0$ and $a_{2}=2.0$ in order to compensate the change in the distribution after taking into account $\left\langle\Delta g_{j}^{+}-\Delta g_{j}^{-}\right\rangle_{\Delta g_{j} \Delta N_{j}}$.

Fig. 8 (a) shows the cumulative distribution of $\Delta g_{j}$ in a log-linear plot. The approximately straight line suggests that the tail follows an exponential distribution which can be well fitted with $P\left(x>\Delta g_{j}\right)=e^{-a\left(x-x_{0}\right) / \Delta \bar{g}}$ where $\Delta \bar{g} \approx 0.12$ is the average of all $\Delta g_{j}$ and the parameters are $a=3.6$ and $x_{0}=0.094$. In the region of the smallest values of $\Delta g_{j} \lesssim x_{0}$ the limited tick sizes of the different 


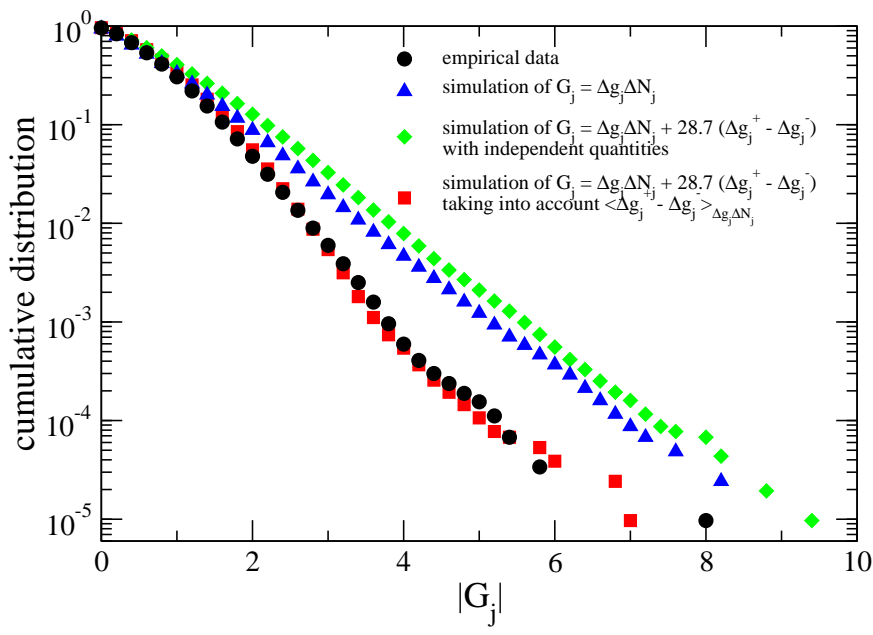

FIG. 9: (Color online) Cumulative distribution of the empirical aggregate return (circles) obtained from ten Nasdaq stocks in comparison with different simulations. A simulation of Eq. 5 (triangles) leads to a reasonable approximation of the empirical data, but it overestimates the probability of large returns. It becomes a little broader if we add the tick return asymmetry $\Delta g_{j}^{+}-\Delta g_{j}^{-}$according to Eq. 7 and simulate independent quantities (diamonds). The simulation (squares) matches the empirical data very well if we incorporate correlations by generating $\Delta g_{j}^{+}-\Delta g_{j}^{-}$according to the conditional expectation value $\left\langle\Delta g_{j}^{+}-\Delta g_{j}^{-}\right\rangle_{\Delta g_{j} \Delta N_{j}}$.

stocks lead to a plateau. In section IV we already found evidence that $\Delta N_{j}$ behaves similarly to uncorrelated data since in Fig. [6 the shuffled data shows almost the same dependence on the rank of the corresponding $\left|G_{j}\right|$. Figure 8 (b) shows that indeed $\Delta N_{j}$ can be well described by a Gaussian noise with mean 0.24 and standard deviation 9.0 .

In order to analyze the accuracy of the approximation given in Eq. [5 we simulate two independent time series according to the fitted functions for $\Delta g_{j}$ and $\Delta N_{j}$ and build the aggregate return $G_{j}$ as the product of $\Delta g_{j}$ and $\Delta N_{j}$. In Figure 9 we can compare the empirically found cumulative distribution of aggregate returns $\left|G_{j}\right|$ (circles) to the results of this simulation (triangles). The simulation of Eq. 5 leads to a reasonable agreement with the actual aggregate return, but it overestimates the probability of large aggregate returns. We note that the parameters of the simulation are completely determined by the empirically found distributions of $\Delta g_{j}$ and $\Delta N_{j}$, so that in this sense the simulation of $\left|G_{j}\right|$ has no free parameters.

In the following, we want to address the remaining deviations of the simulation from the empirical data. Eq. 7 gives an exact formula for $G_{j}$ and provides a good parameterization for the error term which reads

$$
G_{j}-\Delta g_{j} \Delta N_{j}=\frac{2 n_{j}^{+} n_{j}^{-}}{n_{j}}\left(\Delta g_{j}^{+}-\Delta g_{j}^{-}\right)
$$

We find that the term $2 n_{j}^{+} n_{j}^{-} / n_{j}$ has no systematic in- fluence on the aggregate return since it shows almost no dependence on the rank according to the aggregate return. In the following, we thus approximate it by its average value $\left\langle 2 n_{j}^{+} n_{j}^{-} / n_{j}\right\rangle=28.7$, so that the error term is determined by the asymmetries $\Delta g_{j}^{+}-\Delta g_{j}^{-}$in the mean tick return size.

The cumulative distribution of $\Delta g_{j}^{+}-\Delta g_{j}^{-}$is shown in Fig. 8 (c). The main part of the distribution could be well fitted by an exponential function, but in the tail the distribution becomes broader. Thus, we add the term with $\Delta g_{j}^{+}-\Delta g_{j}^{-}$to our simulation by creating a third independent time series according to the empirical distribution of $\Delta g_{j}^{+}-\Delta g_{j}^{-}$. Fig. 9 (diamonds) shows that this leads to an even broader distribution of the aggregate return. Since the difference to the distribution according to Eq. [5 is small, the tick return asymmetry seems to have only a small influence on the aggregate return.

A more accurate agreement with the empirical data can be obtained by taking into account correlations between the quantities involved in the process. The correlation coefficients between them are shown in the following table where the correlations between the absolute values are shown in brackets:

\begin{tabular}{|c|c|c|c|}
\hline & $\Delta N_{j}$ & $\Delta g_{j}^{+}-\Delta g_{j}^{-}$ & $\Delta g_{j} \Delta N_{j}$ \\
\hline$\Delta g_{j}$ & $-0.02(-0.07)$ & $-0.01(0.37)$ & $-0.01(0.34)$ \\
$\Delta N_{j}$ & 1 & $-0.35(0.01)$ & $0.95(0.87)$ \\
$\Delta g_{j} \Delta N_{j}$ & $0.95(0.87)$ & $-0.41(0.02)$ & 1 \\
\hline
\end{tabular}

$\Delta g_{j}$ and $\left|\Delta N_{j}\right|$ show slightly negative correlations which might suggest that people act more cautiously when large tick returns indicate a low liquidity. In these times, traders try not to place too many consecutive orders with the same sign because they know that it could lead to a large price change and increased trading costs. Furthermore, the strong anti-correlations between $\Delta N_{j}$ and $\Delta g_{j}^{+}-\Delta g_{j}^{-}$also indicate cautious traders: If there are large asymmetries, so that e.g. the positive tick returns are much larger than the negative ones, people tend to use the higher liquidity in negative direction so that in these times they sell more often than they buy. For an analysis of the relation between liquidity imbalance and market efficiency, see e.g. [43]. The large correlations between $\Delta g_{j}$ and $\left|\Delta g_{j}^{+}-\Delta g_{j}^{-}\right|$show that we can expect large variations of the tick return in positive and negative direction when the tick return is in general large.

We now want to incorporate correlations in our simulation. The strongest non-trivial correlations appear between $\Delta g_{j} \Delta N_{j}$ and $\Delta g_{j}^{+}-\Delta g_{j}^{-}$including also some of the correlations between $\Delta g_{j}^{+}-\Delta g_{j}^{-}$and $\Delta g_{j}$ as well as $\Delta N_{j}$. However, it turns out that the conditional expectation value $\left\langle\Delta g_{j}^{+}-\Delta g_{j}^{-}\right\rangle_{\Delta g_{j} \Delta N_{j}}$ is non-linear, as seen in Fig. 10 (circles) where it is plotted against $\Delta g_{j} \Delta N_{j}$. 


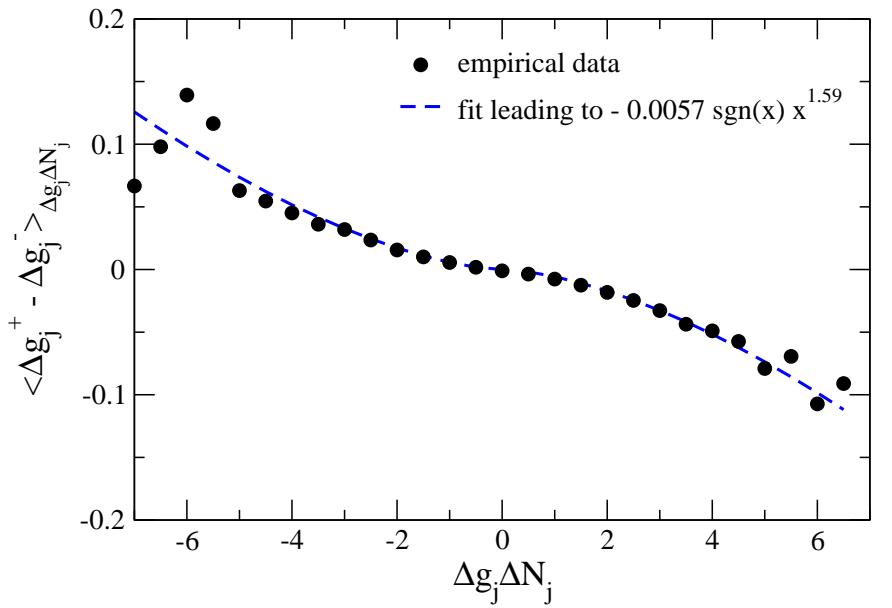

FIG. 10: (Color online) Conditional expectation value $\left\langle\Delta g_{j}^{+}-\Delta g_{j}^{-}\right\rangle_{\Delta g_{j} \Delta N_{j}}$ plotted against $\Delta g_{j} \Delta N_{j}$ (circles), obtained from the data of 10 Nasdaq stocks. A fit leads to $\left\langle\Delta g_{j}^{+}-\Delta g_{j}^{-}\right\rangle_{\Delta g_{j} \Delta N_{j}} \approx-0.0057 \cdot \operatorname{sgn}\left(\Delta g_{j} \Delta N_{j}\right)$. $\left(\Delta g_{j} \Delta N_{j}\right)^{1.59}$ (dashed line). The tick return asymmetry $\Delta g_{j}^{+}-\Delta g_{j}^{-}$is strongly correlated with the mean tick return size $\Delta g_{j}$ and strongly anti-correlated with the number difference $\Delta N_{j}$. Using the conditional expectation value in the simulation incorporates these correlations which allows for the reproduction of the distribution of aggregate returns.

The function can be well fitted by $-\operatorname{sgn}(\mathrm{x}) \alpha|\mathrm{x}|^{\beta}$ with $\alpha=0.0057$ and $\beta=1.59$ (dashed line).

In order to incorporate this conditional expectation value into the simulation, we first create three independent time series for $\Delta g_{j}, \Delta N_{j}$, and $\Delta g_{j}^{+}-\Delta g_{j}^{-}$. Then, for each $j$ we add the conditional expectation value $\left\langle\Delta g_{j}^{+}-\Delta g_{j}^{-}\right\rangle_{\Delta g_{j} \Delta N_{j}}$ to $\Delta g_{j}^{+}-\Delta g_{j}^{-}$, according to the value of $\Delta g_{j} \Delta N_{j}$ for that $j$. This method leads to a different distribution for $\Delta g_{j}^{+}-\Delta g_{j}^{-}$than the initial one, so that we can not anymore generate $\Delta g_{j}^{+}-\Delta g_{j}^{-}$from the unconditional empirical distribution. As a rough approximation, we parameterize this distribution by two exponential functions $e^{-a_{1,2} x / \Delta \bar{g}}$ for $\Delta g_{j}^{+}-\Delta g_{j}^{-} \lessgtr 0.1$. Then, we adapt the factors in the exponent in such a way that the resulting unconditional distribution fits the empirical one (a fit to the empirical distribution yields $a_{1}=8.0$ and $a_{2}=4.8$, for the simulation we use the adapted $a_{1}=9.0$ and $a_{2}=2.0$, compare Fig. 8 (c)). The resulting distribution of $G_{j}$ does not depend very much on the exact values of $a_{1,2}$.

The effect of the correlations represented by the conditional expectation value $\left\langle\Delta g_{j}^{+}-\Delta g_{j}^{-}\right\rangle_{\Delta g_{j} \Delta N_{j}}$ is very large and leads to a cumulative distribution of $\left|G_{j}\right|$ (squares in Fig. 9) very similar to the empirical one (circles). It is worth noting that now the largest events are not anymore necessarily the ones with the largest values of $\Delta g_{j} \Delta N_{j}$. Due to the anti-correlations expressed in $\left\langle\Delta g_{j}^{+}-\Delta g_{j}^{-}\right\rangle_{\Delta g_{j} \Delta N_{j}}$, very large values of $\Delta g_{j} \Delta N_{j}$ can lead to relatively large values of $\Delta g_{j}^{+}-\Delta g_{j}^{-}$of the op- posite sign reducing the aggregate return.

In addition to the distribution of the aggregate return, the simulation does also agree with other properties of the empirical data we found earlier in this paper. In Fig. [3] and 6 we also plotted the data from the simulation against the rank according to the aggregate return $\left|G_{j}\right|$. For $\Delta N_{j}$ the simulation matches the empirical data very well, while in Figure 3 we see that the simulated $\Delta g_{j}$ shows the same dependence on the rank as the empirical data, but it is generally a little larger than the real one except for the largest aggregate returns, which might be due to the cutoff around 0.094 we used in the simulation of the distribution of $\Delta g_{j}$. We also find that the role of $\Delta g_{j}^{+}-\Delta g_{j}^{-}$in determining large aggregate returns is a little overestimated by our simulation, but the simulation covers the main features of the empirical data although we neglected many of the subtle relations between the different quantities.

\section{DISCUSSION AND CONCLUSION}

Our results can be divided into two parts: First, we showed that the movement of stock prices in intervals with a constant number of trades can be understood as a diffusion process with a varying step width. Here, Gaussian fluctuations of the number difference determine the basic price movement, but the non-Gaussian large price changes are due to changes in the tick return size coinciding with large number differences at the same time. The large influence of the tick return size is caused by its autocorrelations assuring that in a 100 tick interval one can find many large tick returns so that the mean value of the tick return can be large. In such intervals, the price change in response to a trade is large, which is referred to as a period of low liquidity. Thus, the diffusion process of stock returns depends largely on fluctuations in the liquidity, in agreement with the findings of previous works [23, 24, 25, 26].

In the second part of this paper, we found that the distribution of aggregate returns can be reasonably approximated by simulating the microscopic quantities mean tick return size and number difference according to their empirically found distributions. A more accurate agreement can be obtained by taking into account asymmetries in the tick return size in positive and negative direction as well as correlations between the different quantities.

To conclude, we found evidence that price fluctuations in intervals with a constant number of trades can be described by a diffusion process with a varying step width. The long-term autocorrelations in the tick return size make sure that periods of low liquidity, where the price change due to a trade is large, last long enough to cause large aggregate returns in intervals with many trades. Our results suggests that the power law distribution of aggregate returns might not be universal but rather depends on a more complicated mechanism which is a combination of the dynamics of the trading frequency, the 
dynamics of the step width and the Gaussian process of the step direction.

\section{Acknowledgment}

I would like to thank Bernd Rosenow for collaboration in the initial phase of the project, many helpful discus- sions, and a critical reading of the manuscript. I also thank Fengzhong Wang for useful conversations.
[1] L. Bachelier, "Théorie de la spéculation" [Ph.D. thesis in mathematics], Annales Scientifiques de l'Ecole Normale Supérieure III-17, 21 (1900).

[2] B. B. Mandelbrot, J. Business 36, 394 (1963).

[3] R. N. Mantegna and H. E. Stanley, Nature (London) 376, 46 (1995).

[4] T. Lux, Applied Financial Econonomics 6, 463 (1996).

[5] P. Gopikrishnan, M. Meyer, L. A. N. Amaral, and H. E. Stanley, European Physical J. B 3, 139 (1998).

[6] U. A. Muller, M. M. Dacorogna, and O. V. Pictet, "Heavy Tails in High-Frequency Financial Data," in $A$ Practical Guide to Heavy Tails, edited by R. J. Adler, R. E. Feldman, and M. S. Taqqu (Birkhäuser Publishers, 1998), p. 83.

[7] V. Plerou, P. Gopikrishnan, L. A. N. Amaral, M. Meyer, and H. E. Stanley, Physical Review E 60, 6519 (1999); P. Gopikrishnan, V. Plerou, X. Gabaix, and H. E. Stanley, ibid. 62, 4493 (2000).

[8] A. Pagan, J. Empirical Finance 3, 15 (1996).

[9] E. F. Fama, J. Business 38, 34 (1965).

[10] R. N. Mantegna and H. E. Stanley, Nature 383, 587 (1996).

[11] R. N. Mantegna and H. E. Stanley, Physica A 239, 255 (1997).

[12] F. M. Longin, The asymptotic distribution of extreme stock market returns, J. Business, 69(3), 383-408 (1996).

[13] E. F. Fama, J. Business 36, 420 (1963).

[14] V. Pareto, Cours d'Economie Politique (Lausanne and Paris, 1897).

[15] P. Lévy, Théorie de l'Addition des Variables Aléatoires (Gauthier-Villars, Paris, 1937).

[16] P. Gopikrishnan, V. Plerou, L. A. N. Amaral, M. Meyer, and H. E. Stanley, Physical Review E 60, 5305 (1999).

[17] P. K. Clark, Econometrica 41, 135 (1973).

[18] R. R. Officer, J. American Statistical Association. 67, 807 (1972).

[19] P. D. Praetz, J. Business 45, 49 (1972).

[20] R. C. Blattberg and N. Gonedes, J. Business 47, 244 (1974).

[21] M. Loretan and P. C. B. Phillips, J. Empirical Finance 1, 211 (1994).

[22] X. Gabaix, P. Gopikrishnan, V. Plerou and H. E. Stanley, Nature (London) 423, 267 (2003).

[23] P. Weber and B. Rosenow, in Proceedings of the Third Nikkei Econophysics Research Workshop and Symposium, The Fruits of Econophysics, Tokyo, November
2004, edited by H. Takayasu (Springer-Verlag, Berlin, 2005), p. 88.

[24] P. Weber and B. Rosenow, Quantitative Finance 6, 7 (2006).

[25] J. D. Farmer, L. Gillemot, F. Lillo, S. Mike, and A. Sen, Quantitative Finance 4(4), 383 (2004).

[26] V. Plerou, P. Gopikrishnan, L. A. N. Amaral, X. Gabaix, and H. E. Stanley, Physical Review E 62(3), R3023R3026 (2000).

[27] C. M. Jones, G. Kaul, M. L. Lipson, Review of Financial Studies, 7(4), 631-651 (1994).

[28] D. Easley, N. M. Kiefer, M. O'Hara, Review of Financial Studies, 10(3), 805-835 (1997).

[29] Z. Ding, C. W. J. Granger and R. F. Engle, J. Empirical Finance 1, 83 (1993).

[30] C. W. J. Granger and Z. Ding Annales d'Economie et de Statistique 40, 67 (1995).

[31] Z. Ding and C. W. J. Granger J. Econometrics 50, 987 (1996).

[32] T. G. Andersen and T. Bollerslev, J. Finance 52, 975 (1997).

[33] Y. Liu, P. Cizeau, M. Meyer, C.-K. Peng, and H. E. Stanley, Physica A 245, 437 (1997).

[34] P. Cizeau, Y. Liu, M. Meyer, C.-K. Peng, and H. E. Stanley, Physica A 245, 441 (1997).

[35] R. Cont, Ph.D. thesis, Universite de Paris XI, 1998 (unpublished); see also e-print cond-mat/9705075

[36] M. Pasquini and M. Serva, Economics Letters 65, 275 (1999).

[37] Y. Liu, P. Gopikrishnan, P. Cizeau, M. Meyer, C.K. Peng, and H. E. Stanley, Physical Review E 60, 1390 (1999).

[38] We analyzed the following companies (ticker symbols): AMAT, BRCD, BRCM, CSCO, INTC, KLAC, MSFT, ORCL, QLGC, SEBL.

[39] J. P. Bouchaud, Y. Gefen, M. Potters, and M. Wyart, Quantitative Finance, 4(2), 176-190 (2004).

[40] F. Lillo and J. D. Farmer, Studies in Nonlinear Dynamics \& Econometrics, 8(3) (2004).

[41] S. Mike and D. Farmer, physics/0509194 (2005).

[42] J. Y. Campbell, A. W. Lo, A. C. MacKinlay, The Econometrics of Financial Markets, Priceton University Press (1997).

[43] J. D. Farmer, A. Gerig, F. Lillo, and S. Mike, physics/0602015 (2006). 